\title{
AVALIAÇÃO DAS FUNÇÕES HEPÁTICA E RENAL DE CÃES EXPOSTOS AO ANTINEOPLÁSICO DOXORUBICINA
}

\author{
$\mathrm{H}$ epatic and renal evaluation of dogs ex posed to antineoplastic dox orubicin
}

\author{
Ana Paula Massae Nakage ${ }^{a}$, Aureo Evangelista Santana ${ }^{b}$ \\ ${ }^{a}$ Médica Veterinária, D outora Professora, Titular do D epartamento de Medicina Veterinária, Centro Universitário “Barão de \\ Mauá", Ribeirão Preto, SP - Brasil, e-mail: apnkg@ hotmail.com

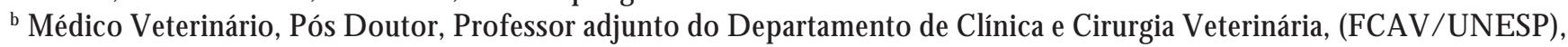 \\ Jaboticabal, SP - Brasil, e-mail: santana@fcav.unesp.br
}

\section{Resumo}

A doxorubicina (A driblastinaâ) é um agente antineoplásico amplamente administrado em O ncologia Humana e Veterinária e eficaz no controle de diversas neoplasias. Porém, sua eficácia é limitada pelo efeito citotóxico não seletivo da doxorubicina. No presente estudo foram utilizados quinze (15) cães adultos sadios, machos e fêmeas, distribuídos em três grupos experimentais: grupo G 1 $(\mathrm{n}=5)$, tratado com solução salina estéril; grupo G $2(\mathrm{n}=5)$, tratado com $20 \mathrm{mg} / \mathrm{m}^{2}$ de doxorubicina e grupo $\mathrm{G} 3(\mathrm{n}=5)$, tratado com $30 \mathrm{mg} / \mathrm{m}^{2}$ de doxorubicina. O s cães receberam solução salina estéril (G1) ou doxorubicina (G 2 e G3) por via intravenosa, a cada 21 dias, até o total de oito aplicações. As avaliações bioquímico-séricas de função hepática e função renal e eletroforéticas foram realizadas seis dias após a exposição à doxorubicina, a intervalos de 21 dias, durante 168 dias. A urinálise foi realizada a cada 30 dias. 0 s animais que vieram a óbito foram necropsiados para caracterização das lesões hepáticas e renais. O s resultados mostraram que a doxorubicina induziu lesões histopatológicas hepáticas e renais, hipo proteinemia, hipoalbuminemia e proteinúria a partir da terceira aplicação da doxorubicina. As ações hepatotóxicas e nefrotóxicas da doxorubicina revelaram-se dose-dependentes, indicando que pacientes submetidos à terapia com doxorubicina devem ser monitorados periodicamente.

Palavras-chave: D oxorubicina; Cães; Fígado; Rins; Toxicidade. 


\begin{abstract}
Doxorubicin (Adriblastinaâ) is an antineoplastic antibiotic agent, extensively used in human and veterinary oncology and useful in the treatment of a variety of tumors. But, its therapeutic efficacy is limited by nonselective cytostatic effect. The study was perfomed on 15 adult healthy dogs of both sexes divided into three groups: group $1(\mathrm{G} 1, n=5)$, injected with $5 \mathrm{ml}$ saline sterile solution; group $2(\mathrm{G} 2, n=5)$, that received $20 \mathrm{mg} / \mathrm{m}^{2}$ of doxorubicin and group $3(\mathrm{G} 3, n=5)$, injected with $30 \mathrm{mg} / \mathrm{m}^{2}$ doxorubicin. The dogs received saline sterile solution (G1) or doxorubicin (G2 and G3) intravenously, every 21 days, until 8 applications were completed. Hepatic and renal evaluation and electrophoretics analisys were determined six days after doxorubicin exposition, every 21 days, for a period of 168 days. Urinalisys was perfomed every 30 days. The animals with spontaneous death, was necropsied to characterize the lesion on liver and kidneys. The results showed that antineoplasic induced hepatic and renal lesions, hipoproteinemia and proteinuria. The hepatotoxicity and nephrotoxicity actions of doxorubicin revealed dose-dependent, indicating that pacients under doxorubicin therapy should be periodically monitored.
\end{abstract}

Keywords: Doxorubicin; Dogs; Liver; Kidneys; Toxicity.

\title{
INTRODUÇÃO
}

A doxorubicina caracteriza-se como um antibiótico antineoplásico antracicleno glicosídico, isolado do fungo Streptomyces peucetius var. caesius (DE MARCO; GAETAMI; SCARPINATO, 1969). A dose usualmente preconizada no tratamento quimioterápico é de $30 \mathrm{mg} / \mathrm{m}^{2}$, a intervalos de 21 dias, por via intravenosa (MOORE, 1993).

O antineoplásico doxorubicina (Adriblastinaâ) tem sido crescentemente aplicado na O ncologia Veterinária, com 0 intuito de proporcionar melhor qualidade de vida aos pacientes neoplásicos. 0 tratamento quimioterápico com doxorubicina tem sido utilizado, na forma de poli ou monoquimioterapia, no controle dos casos de linfomas (SIMON et al., 2008), sarcomas de tecidos moles, nos osteossarcomas (CHUN et al., 2005), nas neoplasias mamárias e em vários carcinomas (KHANNA et al., 1998), porém, a eficácia terapêutica da doxorubicina é limitada em função de suas ações citotóxicas.

A doxorubicina possui três fases de eliminação. A primeira fase representa a rápida distribuição do fármaco para o fígado, pulmões, coração, rins e baço. Na segunda fase ocorre a liberação do fármaco desses órgãos para os compartimentos vasculares e tecidos. D urante a fase final, o fármaco é metabolizado no fígado e excretado na bile e/ ou nas fezes em sete dias (40-50\%), além de ser excretado na urina (5-10\%) (SUSANECK, 1983; HASKELL, 1990; OGILVIE, 1992).

A doxorubicina altera a estrutura do D NA ao ligar-se a este, intercalando entre os pares de bases adjacentes de um filamento e inibindo sua atividade original (MYERS, 1993). Os danos ao DNA também podem ocorrer pela formação de radicais livres, que são responsáveis por severas injúrias celulares nas membranas mitocondriais (LIU; THURMAN, 1992). Haskell (1990) e O gilvie et al. (1991) propõem que os efeitos citotóxicos da doxorubicina são observados durante a fase S (período de síntese de DNA) e G 2 do ciclo celular (período de síntese de RNA e proteína), reduzindo assim o índice mitótico.

O efeito citotóxico da doxorubicina não é seletivo para as células neoplásicas, sendo também deletério para as outras células do organismo (VAN VLEET et al., 1978), como por exemplo, para os hepatócitos (PAPAD O POULOU et al., 1999) e as células glomerulares epiteliais (MANSO UR et al., 1999). D essa maneira, o uso terapêutico desse fármaco tem sido dificultado pelas ações citotóxicas, não seletivas, dele. Segundo Susaneck (1983), a doxorubicina parece ser mais tóxica para o cão do que para o homem. 
O ciclo de óxido-redução da doxorubicina ocorre principalmente no fígado, que é o maior sítio de metabolismo da droga, estando vulnerável a seu efeito tóxico (ODOM et al., 1992). Os principais alvos da doxorubicina no fígado são o DNA (KAMENDULIS; CORCORAN, 1994) e as membranas microssomais dos hepatócitos (JULICHER et al., 1988; FUKUD A et al., 1992). HAUCK et al. (2006) relataram que a administração de doxorubicina causou falência hepática em cães acometidos por sarcomas e carcinomas.

A monitoração dos efeitos hepatotóxicos dos antineoplásicos pode ser realizada através de provas bioquímicas de função hepática, tais como o estudo das aminotransferases, fosfatase alcalina, albumina, bilirrubina sérica e gama-glutamiltransferase (SZ NO L; O HNUMA, 1993).

A ação hepatotóxica pode ser verificada através da histopatologia hepática, na qual pode ser observada a presença de infiltração inflamatória, necrose hepatocelular, fibrose, colestase, lipidose, trombose hepato-portal ou a combinação desses achados (SZNOL; OHNUMA, 1993). As lesões hepáticas induzidas pelo doxorrubiana localizam-se principalmente na região centrolobular (GREVER; GRIESHABER, 1993).

As informações obtidas pelas provas bioquímicas de função hepática e pelas análises histopatológicas do fígado podem ser complementadas pela determinação eletroforética do perfil de proteínas séricas, que pode trazer informações valiosas em relação à capacidade biossintética do fígado e 0 grau de lesão do parênquima hepático (SEVELIUS; ANDERSSON, 1995). Segundo Kaneko (1997), o cão apresenta sete frações proteicas: albumina, alfa-globulinas $\left(\mathrm{a}_{1}\right.$ e $\left.\mathrm{a}_{2}\right)$, beta glo bulinas $\left(\mathrm{b}_{1}\right.$ e $\mathrm{b}_{2}$ ) e gama-globulinas $\left(g_{1}\right.$ e $\left.g_{2}\right)$. 0 perfil do traçado eletroforético e os valores absolutos de cada fração proteica fornecem excelente base para elucidação diagnóstica e estudos adicionais das hepatopatias (SCHALL, 1976).

O s rins são responsáveis pela excreção de cinco a 10\% de doxorubicina introduzida no organismo (OG ILVIE , 1992). Assim, dependendo da dose administrada do antineoplásico, pode haver elevação da taxa do "clearance" renal e presença de nefrotoxicidade (HASKELL, 1990). A administração de doxorubicina promoveu lesões renais em cães acometidos por sarcomas e carcinomas (HAUCK et al., 2006).

A toxicidade renal induzida pela doxorubicina ocorre devido à peroxidação lipídica nas membranas das células epiteliais glomerulares pelos radicais livres oxidativos (CHIARRI et al., 1994; MANSOUR et al., 1999) e devido ao transtorno na permeabilidade seletiva às proteínas do capilar glomerular pelos mediadores inflamatórios de células glomerulares (CHIARRI et al., 1994).

Em adição, verifica-se diminuição do fluxo sanguíneo renal, bem como das taxas de filtração glomerular e reabsorção tubular (HASKELL, 1990; GARNICK, 1993). As alterações da função renal pode ser evidenciada pelos níveis elevados de ureia e creatinina no sangue (GARNICK, 1993).

A nefrotoxicidade da doxorubicina pode induzir a síndrome nefrótica, que é indicada por alterações glomerulares, hipoproteinemia, proteinúria, anasarca, hipertensão, hipercolesterolemia e coagulapatias. (CHIARRI et al., 1994).

A ação da doxorubicina pode ser observada em lesões histopatológicas renais, tais como: glomerulopatia caracterizada por esclerose e vacuolização do glomérulo, deposição de substância hialina sobre a lâmina tubular, atrofia, dilatação tubular, lesões difusas no epitélio tubular com alterações epiteliais degenerativas e regenerativas, inflamação intersticial e fibrose difusa intersticial (BERNS et al., 1995; ZHANG et al., 1996).

A necrose e a apoptose de células epiteliais tubulares têm função importante na patogênese do dano epitelial renal induzido pela doxorubicina (ZHANG et al., 1996; HERMAN et al., 1997). As células mesangiais e fibroblastos produzem, após determinado estímulo, a matriz extracelular de colágeno, que se acumula no mesângio e no interstício, promovendo a glomeruloesclerose e a fibrose intersticial (O KUDA et al., 1992; G HIG GERI et al., 1994).

O escopo principal deste trabalho foi o de mensurar a ação cito tóxica do agente antineoplásico doxorubicina, através do estudo das alterações hepato-renais, avaliadas através de diferentes provas bioquímicas funcionais, análises eletroforéticas de proteínas séricas e análises histopatológicas, com 0 intuito de propiciar um melhor monitoramento de pacientes submetidos ao tratamento quimioterápico com este fármaco. 


\section{MATERIAL E MÉTODOS}

No presente estudo foram utilizados 15 cães adultos sadios, machos e fêmeas, sem raça definida, com peso oscilando entre 15 e $20 \mathrm{Kg}$. Esses cães, oriundos do Canil Central do Hospital Veterinário "Governador Laudo Natel" da FCAV-UNESP, permaneceram alojados em canis individuais, onde receberam ração e água "ad libitum". Antes do início do experimento, todas as parcelas experimentais foram submetidas à avaliação clínica e laboratorial, sendo que todos os cães selecionados para participar do protocolo experimental foram considerados sadios. Portanto, os cães sadios foram distribuídos em três grupos experimentais: grupo G $1(n=5)$, tratado com solução salina estéril; grupo $\mathrm{G} 2(\mathrm{n}=5)$, tratado com $20 \mathrm{mg} / \mathrm{m}^{2}$ de doxorubicina e grupo $\mathrm{G} 3(\mathrm{n}=5)$, tratado com 30 $\mathrm{mg} / \mathrm{m}^{2}$ de doxorubicina. O s cães receberam solução salina estéril (G 1) ou doxorubicina (G 2 e G 3) por via intravenosa, a cada 21 dias, até 0 total de 8 aplicações. Os testes bioquímicos de funcionalidade hepática avaliados foram: alanina aminotransferase (ALT) UV cinética pelo método de Reitman-Frankel modificado, 1957; fosfatase alcalina (FA) PNP cinética pelo método de Roy modificado, 1970; gama-glutamiltransferase (GGT) espectrofotométrica pelo método de Szasz modificado, 1969 e proteína total (PT) colorimétrica pelo método com Biureto. Os testes bioquímicos de funcionalidade renal avaliados foram: ureia (UR) UV cinética pelo método do Diacetil modificado e creatinina (CREA) colorimétrica pelo método de Lustosa - Basques. As amostras de soro para a realização dos testes bioquímicos foram obtidas a partir do sangue total sem anticoagulante e, após sinérese, envasadas e armazenadas adequadamente até o momento das determinações. As dosagens dos parâmetros bioquímicos, acima mencionados, foram realizadas 6 dias após cada administração de solução salina (G 1) ou de doxorubicina (G 2 e G 3), a intervalos de vinte e um (21) dias, durante 168 dias, com 0 auxílio de conjuntos de reagentes ${ }^{1}$, através de leitura cinética, colorimétrica (Labquest) e espectrofotométrica (Espectrofotômetro CELM E - 225D).

As amostras de soro para a realização da eletroforese de proteínas séricas foram obtidas a partir do sangue total sem anticoagulante e, após sinérese, envasadas e armazenadas adequadamente até 0 momento das determinações. A eletroforese de proteínas séricas, realizada 6 dias após cada administração de solução salina (G1) ou doxorubicina (G2 e G3), a intervalos de 21 dias, consistiu, resumidamente, na obtenção do eletroforetograma, através da migração das frações proteicas pelo gel de agarose (albumina, $a_{1}$ e $a_{2}, b_{1}$ e $b_{2}, g_{1}$ e $g_{2}$-globulinas), em um campo elétrico com leituras densitométricas, que forneceram os valores absolutos de cada fração proteica.

As amostras de urina foram obtidas por micção natural e avaliadas, de acordo com as suas características físicas (cor, odor, aspecto e densidade), químicas (pH, presença de nitrito, proteínas, glicose, corpos cetônicos, urobilinogênio e bilirrubinas) e sedimentoscópicas (presença de hemácias, leucócitos, células epiteliais e cilindros), através de análises laboratoriais de rotina, a cada 30 dias.

Os animais que vieram a óbito, durante a etapa experimental, foram submetidos ao estudo anatomopatológico. Foram colhidos fragmentos de fígado e rins, que foram fixados, desidratados, diafanizados, incluídos em parafina, corados com Hematoxilina-Eosina (HE) e Ácido Periódico de Schiff (PAS).

A análise estatística seguiu, para os diferentes parâmetros estudados, um delineamento inteiramente casualizado (D IC), cujos valores médios obtidos foram comparados pelo Teste de Tukey (SNED ECO R; CO CHRAN, 1987), tomando como base a diferença mínima significativa (D MS), com um nível a de probabilidade igual a 5\% (pd"0,05).

\section{RESULTADOS}

Os valores médios dos exames laboratoriais para avaliação da função hepática (alanina aminotransferase-ALT, fosfatase alcalina-FA, gama-glutamiltransferase-G G T, proteínas totais-PT, albumina-ALB, $\mathrm{a}_{1}$-globulina e $\mathrm{a}_{2}$-globulina) e renal (ureia e creatinina-CREA) das parcelas experimentais estão apresentados na Tabela 1 . 
A urinálise não indicou diferenças entre os cães do grupo controle e dos grupos expostos à doxorubicina. Entretanto, os animais tratados com doxorubicina ( $\mathrm{G} 2$ e G 3) revelaram intensa proteinúria, especialmente aqueles que receberam a maior dosagem do quimioterápico (G3).

TABELA 1 - Valores médios dos testes de avaliação da função hepática (ALT, FA, GGT, PT, ALB, $a_{1}$-glob e $a_{2}$-glob) e renal (ureia e CREA) obtidos 6 dias após a administração de solução salina (G 1) ou 20 mg/ $\mathrm{m}^{2}(\mathrm{G} 2)$ e $30 \mathrm{mg} / \mathrm{m}^{2}$ de doxorubicina (G 3), a cada 21 dias durante 168 dias. Jaboticabal (SP), 2008 ( $\mathrm{n=15}$ )

Table 1 - H epatic and renal function evaluation (A L T, FA, G G T, PT, A L B, - glob, $\mathrm{a}_{2}$-glob, ureia, CRE A ) obtained after six days of administration of physiological solution (G 1) or $20 \mathrm{mg} / \mathrm{m}^{2}$ (G 2) and $30 \mathrm{mg} / \mathrm{m}^{2}$ of dox orubicina (G 3), each 21 days during 168 days. Jaboticabal (SP), $2008(n=15)$

\begin{tabular}{|c|c|c|c|c|c|}
\hline & G1 & G2 & G3 & Teste de F (G) & Teste de F (G x T) \\
\hline $\begin{array}{l}\text { ALT } \\
(\mathrm{U} / \mathrm{mL})\end{array}$ & $35,19^{1}$ & 40,86 & 42,81 & $0,33^{\mathrm{NS}}$ & $1,21^{\mathrm{NS}}$ \\
\hline $\mathrm{FA}(\mathrm{U} / \mathrm{L})$ & 47,0 & 38,9 & 47,2 & $1,29^{\mathrm{NS}}$ & $0,90^{\mathrm{NS}}$ \\
\hline GGT (U/L) & $5,33 \mathrm{a}$ & $4,59 \mathrm{ab}$ & $3,19 \mathrm{~b}$ & $4,49 *$ & $0,63^{\mathrm{NS}}$ \\
\hline PT $(g / d L)$ & 7,41 & 7,02 & 6,48 & $6,44^{*}$ & $4,59 * *$ \\
\hline $\operatorname{ALB}(g / d L)$ & $2,62 \mathrm{a}$ & $2,64 \mathrm{a}$ & $2,36 \mathrm{~b}$ & $10,97 *$ & 1,59 NS \\
\hline $\begin{array}{l}a_{1}-g l o b \\
(g / d L)\end{array}$ & 1,05 & 0,93 & 0,82 & $11,09 * *$ & $2,14^{*}$ \\
\hline $\begin{array}{l}a_{2}-g l o b \\
(g / d L)\end{array}$ & 0,61 & 0,68 & 0,71 & $1,82^{\mathrm{NS}}$ & $2,25 *$ \\
\hline (mg/ dL) & 36,1 & 38,1 & 35,7 & $0,12^{\mathrm{NS}}$ & $1,09^{\mathrm{NS}}$ \\
\hline $\begin{array}{l}\text { CREA } \\
(\mathrm{mg} / \mathrm{dL})\end{array}$ & 0,97 & 0,94 & 0,91 & $0,21^{\mathrm{NS}}$ & $2,03 *$ \\
\hline
\end{tabular}

NS - Não significativo

* - Significativo a $5 \%$

** - Significativo a $1 \%$

Valores médios da mesma linha com letras minúsculas iguais não se diferem $(p>0,05)$ e comparam os diferentes grupos $(G)$ para cada tempo de avaliação (T).

ALT = alanina aminotransferase / FA = fosfatase alcalina / GGT = gama-glutamiltransferase / PT = proteínas totais $/$ ALB $=$ albumina $/ a_{1}-g l o b=a_{1}-$ globulina $/ a_{2}-$ glob $=a_{2}-$ globulina $/$ CREA = creatinina .

\section{DISCUSSÃO}

Os achados obtidos nas provas bioquímico-séricas de função hepática, tais como, avaliação das atividades séricas das enzimas hepáticas ALT, FA e GGT revelou que não houve significância entre os grupos nos diferentes momentos de avaliação. Tais resultados discordam daqueles citados por $\mathrm{G}$ rever e G rieshaber (1993), Kamendulis e Corcoran (1994) e Nakage et al. (1999), os quais observaram alterações enzimáticas hepáticas em animais expostos à doxorubicina. Sznol e O hnuma (1993) relataram que a elevação enzimática ocorre somente quando a doxorubicina é administrada em regime poliquimioterápico.

Além disso, foi verificado neste experimento decréscimo estatisticamente significativo $(p<0,01)$ nos níveis séricos de proteínas totais, nos cães expostos à doxorubicina (G 2 e G 3), a partir da terceira administração do antineoplásico. A redução proteica foi mais acentuada nos animais que receberam maior dose de doxorubicina (G 3), sendo dose-dependente. A hipoproteinemia induzida pelo antineoplásico foi reportada por Chiarri et al. (1994) e Mansour et al. (1999). 
Com relação aos resultados da avaliação eletroforética de proteínas séricas, verificou-se que os níveis de albumina dos animais expostos a $30 \mathrm{mg} / \mathrm{m}^{2}$ de doxorubicina (G3) estavam reduzidos significativamente em relação aos níveis dos animais controles (G1) e tratados com $20 \mathrm{mg} / \mathrm{m}^{2}$ do quimioterápico (G2). Tais relatos confirmam a citação de Van Hoesel et al. (1986), na qual a hipoalbuminemia é dose-dependente.

Nos animais que receberam doxorubicina (G2 e G 3) houve redução da fração proteica $\mathrm{a}_{1}$ globulina, caracterizada como proteína de fase aguda negativa e elevação da a-globulina, uma proteína de fase aguda positiva. Segundo a literatura consultada, os níveis reduzidos de a - globulina geralmente ocorrem em distúrbios hereditários (RAVEL, 1995), ou em infecções por E hrlichia canis (HARRUS et al., 1998), portanto não há dados literários que permitam a comparação dos resultados obtidos no presente ensaio. Por outro lado, a elevação da a -globulina pode estar relacionada aos processos inflamatórios de fase aguda, hepatite severa e síndrome nefrótica (KANEKO, 1989), já que foram observadas alterações gastrintestinais importantes, lesões glomerulares, hipoproteinemia e proteinúria nos animais que receberam doxorubicina. A pesar de ter ocorrido interação significativa entre os grupos e momentos para $b_{1}$-globulina, não foi verificado alterações nessa fração globulínica entre os grupos controle (G 1) e tratados com doxorubicina (G 2 e G 3). As demais frações globulínicas $\left(b_{2}, g_{1}\right.$ e $\left.g_{2}\right)$ não apresentaram interação significativa entre os grupos ao longo do período de exposição à droga. A interpretação dos resultados obtidos pela eletroforese de proteínas séricas em gel de agarose pode ter sido prejudicada, devido à grande diversidade proteica para cada subgrupo (TRUMEL et al., 1996).

As alterações histopatológicas hepáticas, independentes da dose e do intervalo de administração de doxorubicina, foram traduzidas por degeneração hepática moderada, inflamação intersticial discreta, fibrose periportal, necrose centrolobular ocasional, infiltração lipídica, figuras mitóticas e congestão vascular difusa, característica da hepatotoxicidade da doxorubicina. Tais injúrias hepáticas, exceto as figuras mitóticas, também foram observadas por Sznol e O hnuma (1993) e Nakage (1997), sendo que estas alterações localizaram-se principalmente na região centrolobular do fígado, corroborando os relatos de G rever e Grieshaber (1993).

Com relação às avaliações bioquímico-séricas e histopatológicas hepáticas, verificou-se que não houve alterações nas atividades das enzimas séricas, porém foi observado hipoproteinemia, hipoalbuminemia e lesões histopatológicas. A ausência de alterações enzimáticas pode ter ocorrido pelo comprometimento hepático moderado, já que a função hepática é afetada, sensivelmente, somente quando mais de $80 \%$ do órgão está lesado. Além disso, os hepatócitos têm elevado poder de regeneração, que pode compensar o tecido destruído. Neste ensaio foram observadas degeneração hepática discreta e algumas figuras mitóticas, que podem indicar regeneração hepática. Portanto, a relação entre as avaliações laboratoriais e histopatológicas do órgão pode ser discreta (CO LES, 1986).

Os resultados obtidos na avaliação bioquímico-sérica para função renal mostraram que a administração de doxorubicina causou interação significativa entre grupos e momentos sobre os níveis de creatinina, porém não provocou elevação nos níveis séricos de ureia e creatinina, confirmando os relatos de Fajardo et al. (1980), G rever e G rieshaber (1993) e Chiarri et al. (1994). Tal fato pode ter ocorrido pela ação do sistema de defesa antioxidante dos rins que diminui a atividade nefrotóxica da doxorubicina, além disso, a alteração dos níveis séricos de ureia e de creatinina ocorre quando, pelo menos, 70\% do tecido renal está lesado (JULICHER et al., 1988).

As lesões renais dos animais tratados com doxorubicina (G2 e G3), independentemente da dose e do intervalo de tratamento, revelaram nefrodistrofia difusa moderada com atrofia e vacuolização glomerular, degeneração e atrofia tubular, necrose coagulativa, presença de cilindros hialinos intraluminais, focos de fibrose, inflamação intersticial e quadro congestivo-hemorrágico, característico do efeito tóxico da droga. Essas injúrias também foram citadas por Berns et al. (1995), Z hang et al. (1996) e Herman et al. (1997).

As características físico-químicas e sedimentoscópicas da urina não apresentaram diferenças entre o grupo controle (G1) e os grupos expostos ao antineoplásico (G2 e G3). Entretanto, em relação à presença de proteínas na urina, verificou-se que os cães tratados, principalmente aqueles expostos à $30 \mathrm{mg} / \mathrm{m}^{2}$ de doxorubicina (G 3), apresentaram proteinúria com maior intensidade, assim como reportado por diversos autores tais como Garnick (1993), BERNS et al. (1995) e Pieroni et al. (1995). 
As avaliações bioquímicas e histopatológicas renais, aliadas à urinálise do tipo I, revelaram que a doxorubicinainduziu lesões glomerulares, que alteraram a permeabilidade dos capilares glomerulares às proteínas e causaram hipoproteinemia, hipoalbuminemia, proteinúria e presença de cilindros hialinos na luz tubular renal.

\section{CONCLUSÃO}

Considerando-se os resultados obtidos no presente ensaio, conclui-se que a doxorubicina induziu hipoalbuminemia e lesões histopatológicas no fígado, evidenciando o seu efeito hepatotóxico. Não houve alterações nas concentrações de ureia e creatinina, porém a doxorubicina causou lesões glomerulares, hipoproteinemia e proteinúria, evidenciando sua nefrotoxicidade. As ações da doxorubicina revelaram-se dose-dependentes, indicando a necessidade de constante monitoração hepática e renal nos pacientes submetidos à quimioterapia com 0 referido antineoplásico.

FONTES DE AQUISIÇÃo: Fundação de Amparo à Pesquisa do Estado de São Paulo (FAPESP).

\section{REFERÊNCIAS}

BERNS, J. S. et al. Severe irreversible renal failure after Ifosfamide treatment. Nature Reviews Cancer, v. 76, p. 497-500, 1995.

CHIARRI, M. G. et al. Involvement of tumor necrosis factor and platelet-activating factor in the pathogenesis of experimental nephrosis in rats. Laboratory Investigation, v. 70, n. 4, p. 449-459, 1994.

CHUN, R. et al. Toxicity and efficacy of cisplatin and doxorubicin combination chemotherapy for the treatment of canine osteosarcoma. Journal of the American Animal H ospital Association., v. 41, n. 6, p. 383-387, 2005.

CO LES, E. H. Liver function. In:

W.B. Saunders Company, 1986. p. $129-15 \overline{0}$.

Veterinary clinical pathology. 4th ed. Philadelphia:

DE MARCO, A.; GAETANI, M.; SCARPINATO, B. Adriamycin (MSC 123, 127): a new antibiotic with antitumor activity. Cancer Chemotherapy Report, v. 53, p. 33-37, 1969.

FAJARD O, L. F. et al. Adriamycin nephrotoxicity. Laboratory Investigation, v. 43, p. 242-253, 1980.

FUKUDA, F. et al. Evaluation of adriamycin-induced lipid peroxidation. Biochemical Pharmacology, v. 44, n. 4, p. 755-760, 1992.

GARNICK, M. B. Urologic complications. In: HOLLAND, J. F. et al. Cancer medicine. Philadelphia: Lea \& Febiger, 1993. v. 2, p. 2323-2331.

G HIG G ERI, G. M. et al. Intact renal albumin downregulates the extracelular matrix expression by mesangial cells and renal fibroblasts in vitro. N ephron, v. 68, p. 353-359, 1994.

GREVER, M. R.; GRIESHABER, C. K. Toxicology by organ system. In: HOLLAND, J. F. et al. Cancer Medicine. Philadelphia: Lea \& Febiger, 1993. v. 1, p. 683-697.

HARRUS, S. et al. Acute blindness associated with monoclonal gammopathy induced by E rlichia canis infection. Veterinary Parasitology, v. 78, n. 31, p. 155-160, 1998.

HA SKELL, C. M. Principles and practice of cancer chemotherapy. In: Philadelphia: Saunders, 1990. p. 21-27.

Cancer treatment.

HAUCK, M. L. et al. Phase I trial of doxorubicin-containing low temperature sensitive liposomes in spontaneous canine tumors. Clinical Cancer Research., v. 12, n. 13, p. 4004-4010, 2006. 
HERMAN, E. H. et al. Comparison of the structural changes induced by doxorubicin and mitoxantrone in the heart, kidney and intestine and characterization of the Fe(III)-mitoxantrone complex. Journal of Molecular and Cellular Cardiology, v. 29, p. 2415-2430, 1997.

JULICHER, R. H. M. et al. The effect of chronic adriamycin treatment on heart kidney and liver tissue of male and female rat. Archieves of Toxicology., v. 61, n. 4, p. 275-281, 1988.

KAMEND ULIS, L. M.; CORCORAN, G. B. DNA as a critical target in toxic cell death: enhancement of dimethylnitrosamine citotoxicity by D NA repair inhibitors. The Journal of Pharmacology Experimental Therapeutics., v. 271, n. 3, p. 1695-1698, 1994.

KANEKO, J. J. Clinical biochemistry of domestical animals. 5th ed. Londres: Academic Press Limited, 1997.

KHANNA, C. et al. Randomized controlled trial of doxorubicin versus dactinomycin in a multiagent protocol for treatment of dogs with malignant lymphoma. Journal of the American Animal H ospital Association., v. 213, n. 7, p. 985-990, 1998.

LIU, Y.; THURMAN, R. G. Potentiation of adriamycin toxicity by ethanol in perfused rat liver. The Journal of Pharmacology Experimental Therapeutics., v. 263, n. 2, p. 651-656, 1992.

MANSO UR, M. A. et al. Effect of captopril on doxorubicin-induced nephrotoxicity in normal rats. Pharmacological Research, v. 39, n. 3, p. 233-237, 1999.

MO ORE, S. A. Recent advances in chemotherapy for nonlymphoid malignant neoplasms. Small Animal Oncology, v. 15, n. 8, p. 1039-1050, 1993.

MYERS, C. Anthracyclines and DNA intercalators. In: HOLLAND, J. F. et al. Cancer medicine. Philadelphia: Lea \& Febiger, 1993. v. 1, p. 764-772.

NAKAGE , A. P. M. Estudo das alterações hematológicas e das provas funcionais hepática e renal, em cães clinicamente normais expostos ao agente antineoplásico doxorubicina (Adriblastina ${ }^{\circledR}$ ), 1997, 46 f. Monografia (G raduação Medicina veterinária) - Faculdade de Ciências Agrárias e Veterinárias, Universidade Estadual Paulista, São Paulo, 1997.

.; et al. Estudo dos efeitos da doxorubicina com relação às funções hepática e renal, em cães sadios. Ars Veterinária, v. 15, n. 1, p. 1-6, 1999.

OD OM, A. L. et al. Biochemical determinants of Adriamycinâ toxicity in mouse liver, heart and intestine. Biochemical Pharmacology, v. 43, p. 831-836, 1992.

OGILVIE, G. K. etal. Weekly administration of low-dose doxorubicyn for treatment of malignant lymphoma in dogs. Journal of the Amenican Animal Hospital Association., v. 18, n. 10, p. 1762-1764, 1991.

O GILVIE, G. K. Cancer chemotherapy in veterinary medicine. In: oncology handbook. Colorado: Colorado State University, 1992. p. $\overline{3} \overline{\overline{6}}-\overline{5} 0$.

O KUDA, S. et al. Albuminuria is not aggravanting factor in experimental focal glomeruloesclerosis and hyalinosis. Journal of Laboratory Clinical Medicine, v. 119, p. 245-253, 1992.

PAPAD OPOULOU, L. C. et al. Structural and functional impairment of mitochondria in adriamycin-induced cardiomyopathy in mice: supression of citochrome c oxidase II gene expression. Biochemical Pharmacology, v. 57, n. 5, p. 481-489, 1999.

PIE RO NI, F. et al. Effects of glycosaminoglycans on the glomerular changes induced by adriamycin. Research in Experimental Medicine, v. 195, p. 373-379, 1995. 
RAVEL, R. Serum proteins. In: 1995. p. 342-368.

. Clinical laboratory medicine. 6th ed. St Louis: Mosby,

SCHALL, W. D. Laboratory diagnosis of hepatic disease. Veterinary Clinical North American, v. 6, p. 679, 1976.

SEVELIUS, E.; ANDERSSO N, M. Serum protein eletrophoresis as a prognostic marker of chronic liver disease in dogs. The Veterinary Record., v. 137, p. 663-667, 1995.

SIMON, D. et al. Efficacy of a continuous, multiagent chemotherapeutic protocol versus a shortterm single-agent protocol in dogs with lymphoma. Journal American of the Veterinary Medical Association, v. 232, n. 6, p. 879-885, 2008.

SNED ECO R, G. W.; COCHRAN, W. G. Statistical methods. 6th ed. Ames: Iowa State University Press, 1987.

SUSANECK, S. J. D oxorubicin therapy in dog. Journal American of the Veterinary Medical Association, v. 182, n. 1, p. 70-72, 1983.

SZNOL, M.; OHNUMA, T. Hepatotoxic effects of cancer and its treatment. In: HOLLAND, J. F. et al. Cancer medicine. Philadelphia: Lea \& Febiger, 1993. p. 2358-2362.

TRUMEL, C. et al. Serum proteins electrophoresis: guidelines for diagnosis evaluation in the dog cat and horse. Revue de Medicine Vétérinaire, v. 147, n. 2, p. 123-130, 1996.

VAN HOESEL, Q. G. C. M. et al. Time-course study on doxorubicin-induced nephropathy and cardiomyopathy in male and female LOU/ M/ Wsl rats: lack of evidence for a causal relationship. Journal of the National Cancer Institute., v. 76, n. 2, p. 299-307, 1986.

VAN-VLEET, J. F. et al. Effect of selenium-vitamin E and adriamycin induced cardiomyopathy in rabbits. American Journal Veterinary Research, v. 9, p. 997-1010, 1978.

ZHANG, J. et al. D oxorubicin-induced apoptosis in spontaneously hypertensive rats: differential effects in heart, kidney and intestine, and inhibition by ICRF-187. Journal of Molecular and Cellular Cardiology, v. 28, n. 9, p. 1931-1943, 1996.

Recebido: 02/ 05/2008 Received: 05/ 02/ 2008

Aprovado: $14 / 05 / 2008$

A pproved: 05/ 14/ 2008 